\title{
Caracterização e Otimização do Tratamento de Efluente Têxtil por Coagulação-Floculação, Utilizando Coagulante Natural Tanino
}

\section{Characterization and Optimization of Treatment of Textile Effluent by Coagulation-Flocculation, Using Natural Coagulant Tannin}

\author{
Osorio Moreira Couto Junior \\ Departamento de Engenharia Química - UEM, Maringá, PR \\ osorio_eq@yahoo.com.br \\ Ana Paula Ströher \\ Departamento de Engenharia Química - UEM, Maringá, PR \\ ana_stroher@hotmail.com \\ Maria Angélica Simões Dornellas de Barros \\ Departamento de Engenharia Química - UEM, Maringá, PR \\ angelica@deq.uem.br \\ Nehemias Curvelo Pereira \\ Departamento de Engenharia Química - UEM, Maringá, PR \\ nehemias@deq.uem.br
}

Resumo: O presente trabalho aborda sobre o tratamento de um efluente de estamparia com um coagulante natural e alternativo na substituição de coagulantes químicos, o tanino, bem como a otimização dos tempos de mistura e sedimentação. Primeiramente, caracterizou-se o efluente bruto e, posteriormente, foram realizados ensaios em jar test envolvendo coagulação, floculação e sedimentação com diferentes tempos de mistura rápida (2 e 5 minutos), mistura lenta (20 e 30 minutos) e repouso (20 e 30 minutos). Variou-se também a dosagem de coagulante (100, 200, 400, 600, e 800 mg. $L^{-1}$ ), para verificar a concentração ótima de tanino no tratamento. Após cada

Recebido em 09-06-2012 - Aceito em 26-09-2012.

\begin{tabular}{|c|c|c|c|c|c|}
\hline RECEN & 14(1) & p. $79-90$ & jan/jun & 2012 & DOI: $10.5777 /$ RECEN.2012.01.05 \\
\hline
\end{tabular}


ensaio, verificou-se o percentual de remoção da cor, turbidez e DQO. Análises complementares como metais, acidez volátil, alcalinidade, sólidos totais e $\mathrm{DBO}_{5}$ foram realizadas para o efluente bruto e tratado para melhor caracterizar o efluente. Os resultados deste trabalho mostraram que a concentração ótima de coagulante corresponde a $400 \mathrm{mg}$. mg. $\mathrm{L}^{-1}$ e os tempos de mistura que proporcionaram as maiores reduções dos parâmetros estudados foram referentes ao ensaio "e", (tempo de mistura rápida $=2$ minutos, tempo de mistura lenta $=20$ minutos e sedimentação $=30$ minutos). As reduções de DQO, cor e turbidez foram respectivamente: 94,81\%, 99,65\%, $99,17 \%$. Os metais, após o tratamento, encontraram-se em concentrações abaixo das estabelecidas pela Resolução CONAMA no $430 / 11$, exceto para o arsênio e mercúrio.

Palavras-chave: coagulante natural; tanino; tratamento efluente.

\begin{abstract}
The present work investigates the treatment of the stamping effluent with natural and alternative coagulant to substitute chemical coagulants, the tannin, and the optimization of the times of mixing and sedimentation. Firstly, it was characterized the raw effluent and then tests were performed jar test involving coagulation, flocculation and sedimentation with different times of faster mixing ( 2 and 5 minutes), slow mixing (20 and 30 minutes) and repose (20 and 30 minutes). It was also varied the dosage of coagulant $\left(100,200,400,600\right.$, and $\left.800 \mathrm{mg} \cdot \mathrm{mg} . \mathrm{L}^{-1}\right)$ to determine the optimal concentration of tannin. After each test, it was found the rate of removal of color, turbidity and COD. Complementary analyzes as metals, volatile acidity, alkalinity, total solids and BOD5 were performed for treated effluent and to better characterize the effluent. The results of this study showed that the optimum concentration of coagulant corresponds to $400 \mathrm{mg}$. $\mathrm{mg} . \mathrm{L}^{-1}$ and times mixing that provided the greatest reductions of the parameters studied were related to the test "e" (faster mixing time $=2$ minutes, slow mixing time $=20$ minutes and sedimentation time $=$ 30 minutes). The reductions of COD, color and turbidity were, respectively, $94.81 \%$, $99.65 \%$ and $99.17 \%$. The metals after treatment were found at concentrations below those set by Resolution CONAMA n. 430/11, except for arsenic and mercury.
\end{abstract}


Key words: coagulant natural; effluent treatment; tannin.

\section{Introdução}

A indústria têxtil apresenta como característica a produção de poluentes nos três estados físicos da matéria, no entanto, o efluente líquido gerado por esse ramo industrial, em virtude do grande volume, requer maior atenção por apresentar grande potencial de danos ao meio ambiente [1].

As elevadas concentrações de DQO (Demanda Química de Oxigênio) nos efluentes líquidos das indústrias têxteis são decorrentes de uma grande carga de compostos orgânicos presentes nesse tipo de efluente [2]. Neste sentido, observa-se que aproximadamente $50 \%$ dos corantes utilizados no setor têxtil acabam sendo descartados junto com o efluente, contribuindo significativamente para o aumento da cor e da concentração da DQO [3].

Nos últimos vinte e cinco anos, a indústria nacional interessou-se não só pela ideia, como efetivamente investiu na pesquisa e desenvolvimento de produtos coagulantes orgânicos biodegradáveis de origem vegetal. Alguns, à base de tanino, são industrializados e comercializados para a clarificação de água com resultados comprovadamente eficientes tanto como agente coagulante quanto como auxiliar de floculação [4].

Os coagulantes à base de tanino são extraídos da casca da acácia negra ou mimosa (Figura 1), planta que foi introduzida no Rio Grande do Sul em 1918, cujos plantios comerciais desenvolveram-se a partir de 1930. A primeira empresa de extração industrial do tanino iniciou-se em 1941.

O uso de biopolímeros extraídos de vegetais no processo de coagulação tem várias vantagens em comparação com os reagentes: (i) a alcalinidade da água não é consumida durante o processo de tratamento; (ii) o lodo gerado após tratamento apresenta tanto um menor volume como se encontra livre de metais pesados quando comparado com o lodo gerado com o uso de reagentes químicos; (iii) estes biopolímeros podem ser originários de plantas locais com fácil processamento, dando um caráter potencial de baixos custos operacionais quando comparados com reagentes químicos, muitas vezes importados [6]. 


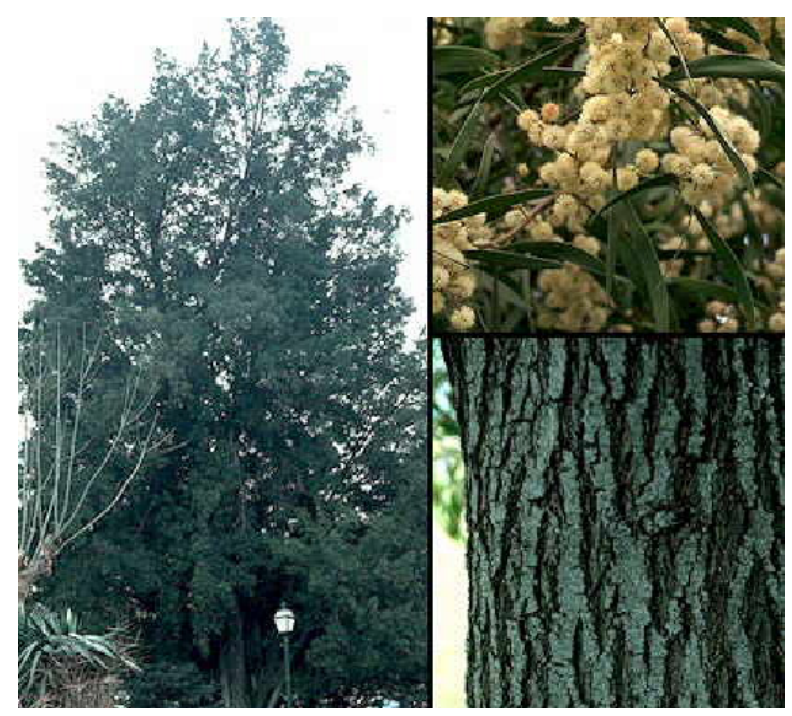

Figura 1. Acácia Negra ou Mimosa [5]

Os despejos de lavanderias industriais são normalmente tratados por meio de processos físico-químicos compostos por coagulação, floculação e sedimentação. Assim, este trabalho apresenta como objetivos a caracterização do efluente de uma estamparia industrial, localizado no município de Floraí, estado do Paraná, e o estudo em laboratório para otimização no tratamento de coagulação-floculação, utilizando o coagulante natural tanino.

\section{Material e métodos}

O efluente utilizado foi coletado em tanque de equalização de uma estamparia industrial. A estação de tratamento da empresa promove o reaproveitamento da água, para lavagem de pisos, com resíduos de corantes provenientes da estamparia.

O coagulante utilizado nos experimentos de coagulação/ floculação/ sedimentação foi o coagulante natural tanino (SG) comercial Tanfloc ${ }^{\circledR}$. Para a realização do tratamento, aferiram-se diferentes quantidades do coagulante em uma balança, realizando em seguida diluições das massas em volumes de $20 \mathrm{~mL}$ do efluente, para ser finalmente adicionados nos béqueres.

Foram realizadas seis coletas do efluente na estamparia que foram encaminhadas para o Laboratório de Processo e Separação (LPS) da Universidade Estadual de 
Maringá, armazenadas em embalagens de 15 litros e conservadas sob refrigeração à temperatura de $15^{\circ} \mathrm{C}$, aproximadamente.

Os ensaios de coagulação/floculação/sedimentação foram realizados à temperatura ambiente em equipamento Jar Test, marca Milan, modelo JT 101/6 de seis provas (Figura 2), com regulador de rotação das hastes misturadoras. Os experimentos consistiram na adição de diferentes dosagens do coagulante $(100,200,400,600$ e 800 mg. $\left.\mathrm{L}^{-1}\right)$ em amostras $(500 \mathrm{~mL})$ de efluente em cada um dos béqueres de prova (500 $\mathrm{mL}$ ). Para encontrar a faixa de concentração para realizar os ensaios, adotou-se como parâmetro inicial a concentração adicionada no processo de tratamento empregado na estamparia e variaram-se as concentrações (inferiores e superiores).

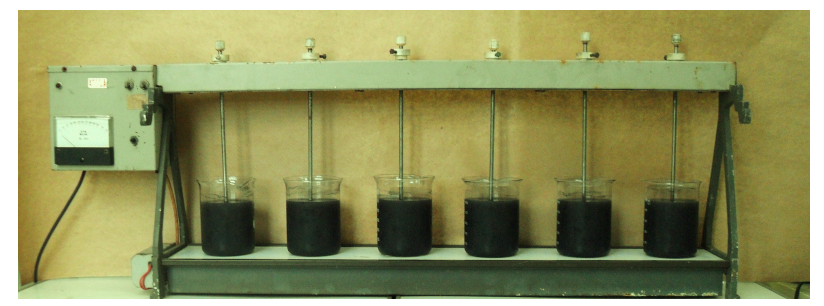

Figura 2. Equipamento Jar Test Milan, modelo JT 101/6

A velocidade de mistura rápida foi fixada em $90 \mathrm{rpm}$, enquanto a de mistura lenta em $35 \mathrm{rpm}$, conforme valores utilizados na estamparia. Variou-se o tempo de mistura rápida (TMR) em 2 e 5 minutos, tempo de mistura lenta (TML) em 20 e 30 minutos, e tempo de sedimentação (SED) em 20 e 30 minutos, de acordo com o planejamento experimental apresentado na tabela 1 .

Tabela 1. Variação dos tempos de mistura rápida, lenta e sedimentação

\begin{tabular}{llll}
\hline Ensaios & TMR (min) & TML (min) & SED (min) \\
\hline $\mathrm{a}$ & 5 & 30 & 30 \\
$\mathrm{~b}$ & 5 & 20 & 30 \\
$\mathrm{c}$ & 5 & 20 & 20 \\
$\mathrm{~d}$ & 2 & 30 & 30 \\
$\mathrm{e}$ & 2 & 20 & 30 \\
$\mathrm{f}$ & 2 & 20 & 20 \\
\hline
\end{tabular}

O sobrenadante obtido após o tratamento foi coletado em cada béquer e seguiu 
para análise dos parâmetros. Assim, pode-se verificar a eficiência de remoção pela comparação dos resultados obtidos com o efluente bruto.

As análises de cor, turbidez e DQO foram realizadas com o auxílio de espectrofotômetro HACH, nos comprimentos de onda, respectivamente: 455, 860 e 600 $\mathrm{nm}$. $\mathrm{O} \mathrm{pH}$ das amostras foi determinado utilizando-se um pHmetro digital, marca Digimed, modelo DM 22. Para melhor avaliar a eficiência do tratamento em estudo, realizaram-se análises complementares de alcalinidade, acidez volátil, $\mathrm{DBO}_{5}$ (Demanda Bioquímica de Oxigênio) sólidos totais e metais. Todas as análises foram realizadas, conforme metodologia descrita no Standard Methods for the Examination of Water and Wastewater [7].

\section{Resultados}

\subsection{Características do efluente bruto}

A caracterização do efluente bruto seguiu os mesmos padrões de análises do efluente tratado com o coagulante natural após a coagulação/floculação. A tabela 2 apresenta a caracterização do efluente em cada uma das coletas.

Tabela 2. Caracterização das amostras coletadas na estamparia

\begin{tabular}{lllll}
\hline Coletas & DQO $\left(\mathrm{mg.L}^{-1} \mathrm{O}_{2}\right)$ & Cor $($ units Pt Co) & Turbidez (FAU) & pH \\
\hline 1 & 2659,73 & 14175 & 5082 & 7,49 \\
2 & 1410,3 & 7392 & 1722 & 7,45 \\
3 & 6977,6 & 31185 & 7902 & 7,19 \\
4 & 5317,2 & 19311 & 4998 & 7,19 \\
5 & 4301 & 15620 & 4042 & 7,45 \\
6 & 4589,1 & 16667 & 4313 & 7,35 \\
\hline
\end{tabular}

$\mathrm{Na}$ tabela 2, observa-se grande variação dos parâmetros DQO, cor e turbidez, ao longo das coletas. Além disso, nota-se que esses parâmetros encontram-se elevados, fato que pode ser explicado pela grande quantidade de insumos, corantes e sujidades ao longo do processo de estampagem. Para os valores de $\mathrm{pH}$, não houve variações significativas pelo fato da coleta ser realizada no tanque de equalização, onde o efluente é ajustado em pH neutro. 
$\mathrm{Na}$ tabela 3, encontram-se parâmetros complementares avaliados para o efluente bruto e os valores limites, estipulados pela Resolução CONAMA no 430/11, para lançamento em corpos receptores.

\begin{tabular}{|c|c|c|}
\hline Descrição & Resultados & CONAMA \\
\hline Acidez Volátil (mg. $\left.\mathrm{L}^{-1}\right)$ & 295 & - \\
\hline Alcalinidade $\left(\mathrm{mg} . \mathrm{L}^{-1}\right)$ & 368 & - \\
\hline $\mathrm{DBO}_{5}\left(\mathrm{mgO} \cdot \mathrm{L}^{-1}\right)$ & 818 & - \\
\hline Sólidos Totais $\left(\mathrm{mg} . \mathrm{L}^{-1}\right)$ & 5702 & - \\
\hline Arsênio (mg. $\mathrm{L}^{-1}$ ) & 2,194 & 0,5 \\
\hline Bário (mg. $\left.\mathrm{L}^{-1}\right)$ & 3,364 & 5 \\
\hline Chumbo (mg. $\left.\mathrm{L}^{-1}\right)$ & 0,642 & 0,5 \\
\hline Cromo $\left(\mathrm{mg} . \mathrm{L}^{-1}\right)$ & 0,015 & 0,5 \\
\hline Ferro (mg. $\left.\mathrm{L}^{-1}\right)$ & 1,543 & 15 \\
\hline Prata (mg. $\left.\mathrm{L}^{-1}\right)$ & 0,138 & 0,1 \\
\hline Manganês (mg. $\left.\mathrm{L}^{-1}\right)$ & 1,529 & 1 \\
\hline Mercúrio (mg. $\mathrm{L}^{-1}$ ) & 0,035 & 0,01 \\
\hline Níquel (mg. $\left.\mathrm{L}^{-1}\right)$ & 0,289 & 2 \\
\hline Zinco (mg. $\left.\mathrm{L}^{-1}\right)$ & 0,652 & 5 \\
\hline
\end{tabular}

Nota-se que parte dos metais avaliados encontra-se abaixo do limite estabelecido pela Resolução CONAMA n ${ }^{\circ}$ 430/11, (bário, cromo, ferro, níquel e zinco), estando os demais metais (arsênio, chumbo, prata, manganês e mercúrio) acima do limite, devendo-se atentar para as referentes concentrações após o tratamento.

\subsection{Experimentos de coagulação/floculação/sedimentação}

$\mathrm{Na}$ tabela 4, estão apresentados os valores de $\mathrm{pH}$ para as concentrações adicionadas em cada um dos ensaios da tabela 1 .

A tabela 4 mostra que, conforme o aumento da concentração do coagulante, ocorreu uma leve diminuição do $\mathrm{pH}$, porém esses decaimentos não são significativos visto que o coagulante natural em estudo possui estabilização em termos desse parâmetro. Estudos apresentados na literatura têm mostrado que o tanino é importante ao ser utilizado, principalmente, para águas de abastecimento ou ainda em efluentes industriais que possuem $\mathrm{pH}$ entre 6,0 e 8,0 [8]. 
Revista Ciências Exatas e Naturais, Vol.14, n 1, Jan/Jun 2012

Tabela 4. Verificação do pH após coagulação/floculação

\begin{tabular}{cllllll}
\hline & \multicolumn{7}{c}{ Ensaios } \\
\hline Concentração $\left(\mathrm{mg}^{-\mathrm{L}^{-1}}\right)$ & $\mathrm{a}$ & $\mathrm{b}$ & $\mathrm{c}$ & $\mathrm{d}$ & $\mathrm{e}$ & $\mathrm{f}$ \\
\cline { 2 - 7 } & $\mathrm{pH}$ & $\mathrm{pH}$ & $\mathrm{pH}$ & $\mathrm{pH}$ & $\mathrm{pH}$ & $\mathrm{pH}$ \\
\hline 100 & 6,7 & 6,7 & 6,8 & 6,8 & 6,7 & 7 \\
& 8 & 3 & 5 & 7 & 4 & 1 \\
200 & 6,77 & 6,69 & 6,8 & 6,85 & 6,7 & 6,86 \\
& 7 & 9 & 8 & 5 & 7 & 6 \\
400 & 6,81 & 6,64 & 6,79 & 6,84 & 6,66 & 6,66 \\
& 1 & 4 & 9 & 4 & 6 & 6 \\
600 & 6,73 & 6,63 & 6,67 & 6,74 & 6,58 & 6,06 \\
& 3 & 3 & 7 & 4 & 8 & 6 \\
800 & 6,66 & 6,58 & 6,66 & 6,64 & 6,52 & 5,97 \\
& 6 & 8 & 6 & 4 & 2 & 7 \\
\hline
\end{tabular}

A tabela 5 apresenta a porcentagem de redução dos parâmetros em estudo após o tratamento proposto, de acordo com a concentração ótima de coagulante.

Tabela 5. Percentual de remoção e concentração ótima para o coagulante

\begin{tabular}{lcccc}
\hline Ensaio & Concentração Ótima $\left(\mathrm{mg.L}^{-1}\right)$ & \multicolumn{3}{c}{ Remoção(\%) } \\
\hline & & DQO & Turbudez & Cor \\
\cline { 2 - 5 } $\mathrm{a}$ & 400 & 93,22 & 99,43 & 98,86 \\
$\mathrm{~b}$ & 400 & 93,77 & 98,6 & 98,79 \\
$\mathrm{c}$ & 400 & 92,14 & 99,55 & 99,19 \\
$\mathrm{~d}$ & 400 & 88,52 & 98,68 & 98,98 \\
$\mathrm{e}$ & 400 & 94,81 & 99,65 & 99,17 \\
$\mathrm{f}$ & 400 & 86,69 & 99,16 & 98,99 \\
\hline
\end{tabular}

De acordo com os resultados apresentados na tabela 5 , os melhores tempos de mistura foram os referentes ao ensaio " $\mathrm{e}$ ", com concentração de $400 \mathrm{mg} . \mathrm{L}^{-1}$ e percentual de remoção de 94,81\%, 99,65\% e 99,17\% para DQO, turbidez e cor, respectivamente.

A figura 3 apresenta a eficiência na redução dos parâmetros DQO, cor e turbidez para cada uma das concentrações do coagulante tanino, referente ao ensaio "e".

Para o efluente bruto oriundo da estamparia, cuja média da DQO é de $4500 \mathrm{mgO}_{2} \cdot L^{-1}$, a melhor remoção para DQO, cor e turbidez, de acordo com a figura 3 , foi obtida utilizando a dosagem de $400 \mathrm{mg} . \mathrm{L}^{-1}$ de coagulante tanino. 
COUTO JUNIOR, O. M. et al

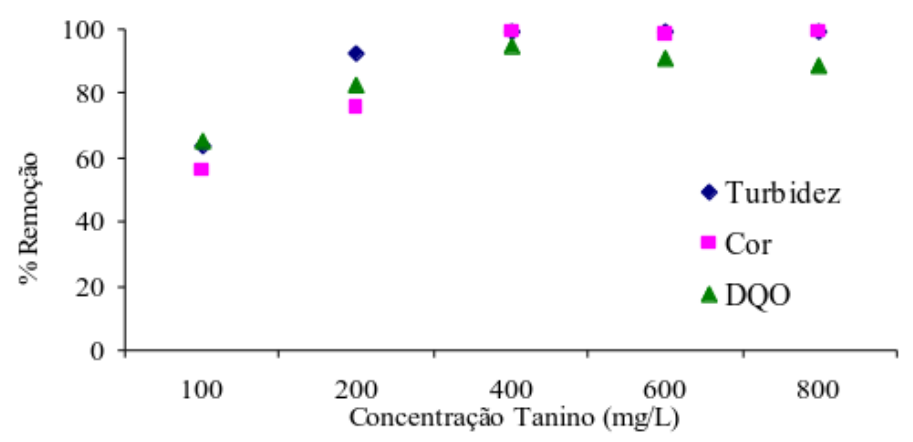

Figura 3. Percentual de redução dos parâmetros DQO, cor e turbidez

$\mathrm{Na}$ tabela 6, estão apresentadas as características físico-químicas do efluente tratado com o tanino no processo de coagulação/floculação/sedimentação e os valores exigidos pela Resolução CONAMA n ${ }^{\circ}$ 330/11.

Tabela 6. Valores médios das características físico-químicas após o tratamento realizado com as condições ótimas determinadas

\begin{tabular}{|c|c|c|}
\hline Descrição & Resultados & CONAMA \\
\hline Acidez Volátil (mg.L $\left.{ }^{-1}\right)$ & 120 & - \\
\hline Alcalinidade $\left(\mathrm{mg} \cdot \mathrm{L}^{-1}\right)$ & 220 & - \\
\hline $\mathrm{DBO}_{5}\left(\mathrm{mgO} 2 . \mathrm{L}^{-1}\right)$ & 60 & - \\
\hline Sólidos Totais $\left(\mathrm{mg} . \mathrm{L}^{-1}\right)$ & 2444 & - \\
\hline Arsênio $\left(\mathrm{mg} \cdot \mathrm{L}^{-1}\right)$ & 0,952 & 0,5 \\
\hline Bário (mg. $\left.\mathrm{L}^{-1}\right)$ & 1,1 & 5 \\
\hline Chumbo (mg. $\left.\mathrm{L}^{-1}\right)$ & 0,349 & 0,5 \\
\hline Cromo $\left(\mathrm{mg} . \mathrm{L}^{-1}\right)$ & 0,011 & 0,1 \\
\hline Ferro (mg. $\mathrm{L}^{-1}$ ) & 0,576 & 15 \\
\hline Prata $\left(\mathrm{mg} . \mathrm{L}^{-1}\right)$ & 0,021 & 0,1 \\
\hline Manganês (mg. $\mathrm{L}^{-1}$ ) & 0,123 & 1 \\
\hline Mercúrio (mg.L $\mathrm{L}^{-1}$ ) & 0,011 & 0,01 \\
\hline Níquel (mg. $\left.\mathrm{L}^{-1}\right)$ & 0,114 & 2 \\
\hline Zinco (mg. $\left.\mathrm{L}^{-1}\right)$ & 0,253 & 5 \\
\hline
\end{tabular}

Nota-se que os valores dos metais encontram-se dentro dos valores estabelecidos pela resolução, exceto para o arsênio e mercúrio. Para essas análises, utilizou-se a melhor dosagem do coagulante de $400 \mathrm{mg} \cdot \mathrm{L}^{-1}$. O tanino oferece condições favoráveis 
para a remoção de metais de águas com características ácidas [9]. De uma forma geral, observa-se, na tabela 6 , que todos os parâmetros apresentaram uma remoção alta com o uso do coagulante tanino.

A comparação visual entre o efluente bruto e o efluente tratado com as condições ótimas determinadas (ensaio "e" e concentração $400 \mathrm{mg} \cdot \mathrm{L}^{-1}$ ) é apresentada na figura 4.

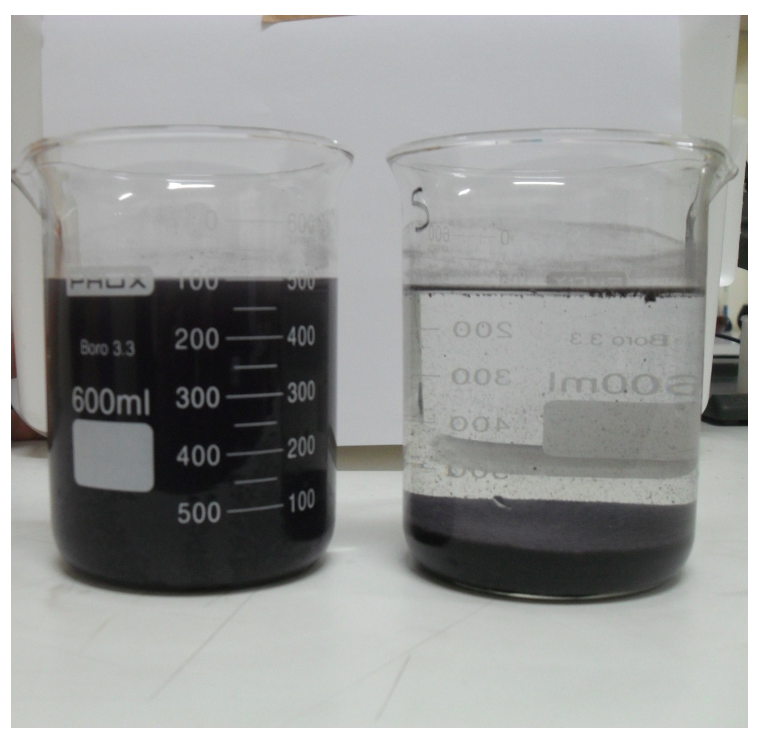

Figura 4. Efluente bruto e efluente tratado com tanino (a direita), com concentração de $400 \mathrm{mg} \cdot \mathrm{L}^{-1}$

O lodo formado pelo uso de coagulantes naturais, como o tanino, é relativamente mais denso e fácil de ser desidratado, facilitando assim, o seu manuseio e a sua disposição, quando comparado ao lodo oriundo do uso de coagulantes químicos [10].

\section{Conclusão}

Para os metais do efluente bruto analisados, a concentração encontra-se abaixo do limite estabelecido pela Resolução CONAMA no 430/11, exceto para o arsênio, chumbo, prata, manganês e mercúrio.

O coagulante em estudo apresentou o melhor percentual de remoção para a concentração de $400 \mathrm{mg} . \mathrm{L}^{-1}$ e para os tempos de mistura referentes ao ensaio "e”, (TMR 
$=2$ minutos, $\mathrm{TML}=20$ minutos, e $\mathrm{SED}=30$ minutos). As reduções de $\mathrm{DQO}$, cor e turbidez foram respectivamente: $94,81 \%, 99,65 \%, 99,17 \%$.

Para a acidez volátil, alcalinidade, $\mathrm{DBO}_{5}$ e sólidos totais, o tanino demonstrou ser eficiente, diminuindo a concentração desses parâmetros no efluente após sua aplicação no tratamento.

Os teores de metais investigados, presentes no efluente tratado com o coagulante natural, foram menores do que os teores estabelecidos pela Resolução CONAMA n ${ }^{\circ}$ 430/11, exceto para arsênio e mercúrio.

\section{Referências}

[1] COLLINS, A. G.; GRASSO, D.; FARVARDIN, M. R. Evaluating preozonation as an aid to coagulation flocculation processes. Proceedings of Ninth Ozone World Congress. June 3-9, New York, USA; 1989.

[2] SIQUARA, L. O. A influência do íon cloreto na determinação da demanda química de oxigênio, Rev Têxtil, São Paulo: R. da Silva Haydu e Cia Ltda, 1994

[3] O’NEILL, C., HAWKES, F.R., HAWKES, D.L., LOURENÇO, N.D., PINHEIRO, H.M., DELEE, W. Colour in Textile Effluents - Sources, Measurements, Discharge Consents and Simulation: a Review. J Chem Technol Biot, v. 74, p.1009-1018, 1999.

[4] BARRADAS, J. L., TANINO Uma solução ecologicamente correta: Agente floculante biodegradável de origem vegetal no tratamento de água, Novo Hamburgo - RS, Brasil, 2004.

[5] CANAZIO, A. SÁNCHEZ. J. M.. Arboles Ornamentales. Disponível em http://www.arbolesornamentales.es/nombreslatinos.htm. Acesso em jun/2012.

[6] SILVA, J.; GOMES, L.P.; DECUSATI, O.; LAMB, L.H. Aplicação de floculante vegetal no tratamento de águas, IV Simpósio Internacional de Qualidade Ambiental, Porto Alegre, RS, 2004. 
Revista Ciências Exatas e Naturais, Vol.14, n 1, Jan/Jun 2012

[7] APHA - American Public Health Association, Standard Methods for the Examination for Water and Wastewater, $19^{t h}$ ed., AWWA, WPCF, Washington, D.C.; 1995.

[8] CRUZ, J. G. H. Alternativas para aplicação de coagulante vegetal à base de tanino no tratamento de efluente de uma lavanderia industrial. Dissertação de Mestrado, Universidade Federal do Rio Grande do Sul, Porto Alegre, 2004.

[9] RICHTER, C. A.; AZEVEDO NETTO, J. Tratamento de água. Tecnologia atualizada, Edgard Blücher, São Paulo, 2003.

[10] ZHAN, X. M.; ZHAO, X. Mechanism of lead adsorption from aqueous solutions using and adsorbent synthesized from natural condensed tannin, Water Res v. 37, p $3905-3912,2003$. 